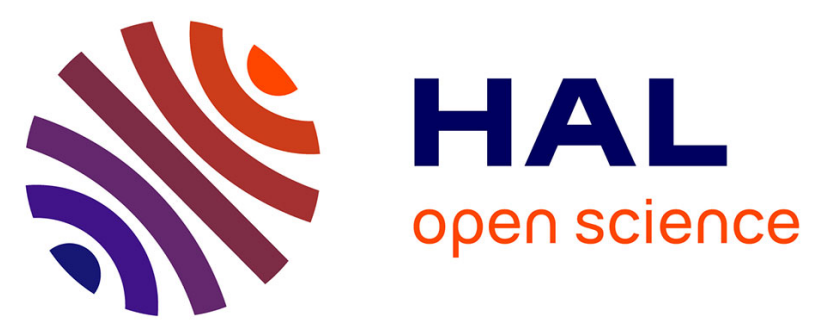

\title{
SEMBLE-T-IL CONVENABLE DE MODIFIER LES MÉTHODES DE TRAVAIL DES DEPOTS CENTRALISATEURS DE LAIT DE FEMME EXIGÉES ET UTILISÉES JUSQU'A PRÉSENT?
}

\author{
K. Kayser
}

\section{To cite this version:}

K. Kayser. SEMBLE-T-IL CONVENABLE DE MODIFIER LES MÉTHODES DE TRAVAIL DES DEPOTS CENTRALISATEURS DE LAIT DE FEMME EXIGÉES ET UTILISÉES JUSQU'A PRÉSENT?. Le Lait, 1953, 33 (329_330), pp.621-624. hal-00928101

\section{HAL Id: hal-00928101 https://hal.science/hal-00928101}

Submitted on 1 Jan 1953

HAL is a multi-disciplinary open access archive for the deposit and dissemination of scientific research documents, whether they are published or not. The documents may come from teaching and research institutions in France or abroad, or from public or private research centers.
L'archive ouverte pluridisciplinaire HAL, est destinée au dépôt et à la diffusion de documents scientifiques de niveau recherche, publiés ou non, émanant des établissements d'enseignement et de recherche français ou étrangers, des laboratoires publics ou privés. 


\title{
BIBLIOGRAPHIE
}

[1] Prévot et Thouvenot. Ann. Inst. Past., 1952, 83, 180.

[2] Prévot. Ann. Inst. Past., 1948, 75, 571.

[3] Prévot, Saissac et Callame. Ann. Inst. Past., 1950, 79, 93.

[4] Paquet et GaUvin. Canad. Journal Health., 1953, 44, 92.

[5 ]Prévot et Moureav. Ann. Inst. Past., 1952, 82, 13.

[6] PrÉvot. La Nature, 1952, no 3.205, p. 147.

[7] Ratbatd, Mémoire de Concours.

\section{SEMBLE-T-IL CONVENABLE DE MODIFIER LES MÉTHODES DE TRAVAIL DES DÉPOTS CENTRALISATEURS DE LAIT DE FEIMIME EXIGÉES ET UTILISÉES JUSQU'A PRÉSENT?}

\author{
par
}

\section{K. KAYSER}

L'opinion des pédiatres allemands que le lait de femme est la meilleure source de nourriture pour les nouveau-nés et les nourrissons fait toujours autorité, et les laits humanisés de vaches n'ont pas encore prouvé d'une façon convaincante leur supériorité.

Aussi longtemps qu'il en sera ainsi, les dépôts centralisateurs de lait de femme (F.M.S.) ont leur raison d'être dans la lutte contre la mortalité infantile.

La fondatrice des F.M.S., M. E. KAYSER, a pourtant toujours bien répété que le lait de femme stérilisé des dépôts centralisateurs se range derrière le lait tété, et qu'il est malgré les affirmations de ses adversaires meilleur que le lait de vache bouilli et ses mélanges. Il a été prouvé par nos essais et ceux d'autres expérimentateurs sur des jumeaux univitellins que l'affirmation des adversaires du lait de femme que celui-ci est nuisible aux enfants n'est pas justifiée.

Pour que le lait des F.M.S. ne nuise pas aux enfants, M. E. KAYSER estime que trois conditions doivent être remplies dans la livraison du lait de femme aux F.M.S.

a) Le lait de femme ne doit subir aucune sorte d'addition non voulue (en premier lieu, pas de lait étranger, d'eau et analogues);

b) Le lait ne doit avoir subi aucune espèce de décomposition (détermination de l'acidité et du $p \mathrm{H}$ ) ;

c) Le lait doit être traité au F.M.S. de telle façon qu'aucune modification de ce lait ne puisse nuire aux enfants.

Pour remplir ces trois eonditions, M. E. KAYSER a perfectionné les méthodes les plus diverses de recherches et de conservation du

(1) Zentralblatt für Gynäkologie, 1952, fascicule 12. 
lait, auxquelles chaque portion isolée de lait de femme livrée doit être soumise avant son utilisation et a établi ainsi un traitement obligatoire supplémentaire.

La F.M.S. d'Erfurt ne peut pas comprendre que la F.M.S. de Greifswald semble perdre complètement de vue les conditions fondamentales qui y ont été aussi utilisées, et que d'après les déclarations de Russ dans "Kinderärtzlichen Praxis " elle laisse la clinique infantile de Greifswald rassembler, traiter et distribuer le lait d'une façon qui, malgré les bons résultats indiqués par Russ, nous paraît être dangereuse au plus haut degré.

Le ramassage et la conservation en bouteilles d'un litre rendent impossible que les rations de lait soient entièrement examinées individuellement; des qualités de lait de valeurs différentes sont done nécessairement mélangées ensemble, ceci concernant spécialement les degrés d'acidité différents, de même qu'éventuellement les impuretés et les falsifications éventuelles.

On n'évite pas cela. Et la paysanne de Rügen qui, pendant l'écoulement ou le pompage entend soudainement que ses petits cochons ou ses poussins, etc., sont en danger quelconque laisse le pompage en plan, afin d'éloigner le danger, oublie le lait extrait qu'elle retrouvera quelques heures après recouvert de mouches et qu'elle versera alors sans réflexion dans la bouteille d'un litre dans laquelle se trouve peut-être du lait irréprochable. En effet, elle touche 8,50 D. M. par litre!

Nous tenons en outre pour très grave de mettre entre les mains des distributrices une bouteille d'un litre et les eitrettes (1). A la F.M.S. d'Erfurt qui s'assure toujours et toujours sur place de la façon dont le lait est rassemblé et conservé, dans laquelle le lait est livré quotidiennement, on constate constamment des bévues faites par les distributrices opérant par la méthode d'Erfurt. Infiniment souvent, des bouteilles individuelles de 200 grammes du même jour de la même distributrice sont contestées au point de vue qualité après recherches. Et de plus laisser le lait de femme des journées ensuite chez la paysanne? Lui laisser le soin de faire le mélange aveo les citrettes ? Quand comme directeur d'une clinique publique d'accouchement on peut toujours constamment constater que, même avec un "soi-disant " contrôle des falsifications, se produisent dans le lait obtenu par pression, on peut déjà en tirer la conclusion que même les femmes mecklembourgeoises-poméraniennes ne sont pas des anges. "Plus on en a, plus on veut." La femme ręoit 8,50 D.M. par litre.

C'est là la malédiction du paiement du lait. A notre avis, le lait

(1) Comprimós dont la substance active est l'acide citrique. 
de femme est impayable, une "largesse" pour laquelle les mères doivent assurément recevoir une compensation afin de pouvoir remplacer entièrement les calories contenues dans le lait donné et qui sont utilisées par synthèse.

Jusqu'à quel point la nature du lait de femme additionné d'un pourcentage relativement élevé d'acide citrique est-elle modifiée échappe à ma connaissance. L'affirmation de Russ que par la conservation du lait à l'aide des citrettes, la "cuisson dénaturatrice " sera évitée est, à mon avis, à munir d'un point d'interrogation. Son affirmation n'est pas en tous cas démontrée par le fait que les enfants nourris au lait de citrettes se développent bien.- Ceci se produit aussi dans toutes les cliniques d'enfants qui, depuis des années, et même des dizaines d'années, reçoivent du lait de femme stérilisé et aussi chez les nourrissons qui ont reçu du lait de femme stérilisé de nous et d'autres dépôts centralisateurs. La demande n'en serait pas si importante si le préjudice répété invoqué par les adversaires du lait de femme stérilisé existait.

Bernheim-Carrer, M. E. KAySER et d'autres, qui se sont penchés sans parti pris sur les dommages que pourrait causer au lait de femme la cuisson, ont, en tous cas, par leurs essais sur des jumeaux univitellins rassemblé des résultats opposés à ceux des adversaires. Ceux-ci sont à consulter dans la littérature soientifique.

Malgré cela, il serait toutefois désirable qu'une conservation du lait de femme soit possible, qui éviterait indubitablement les modifications du lait produites par la stérilisation, malgré toutes les sécurités qu'offrent les recherches exigées dans les dépôts centralisateurs et qu'offre aussi la stérilisation.

Quand les préparations de lait humanisé répondront réellement à ce qui leur est actuellement attribué, les F.M.S. n'auront plus de raison d'être et le différend sur la façon de conserver le lait disparaîtra aussi. Nous n'aurions plus alors à forcer ainsi un travail silencieux, comme cela se produit assez souvent à la elinique régionale pour femmes d'Erfurt où 96 à $98 \%$ des nouveau-nés ne sont nourris pendant les dix premiers jours de leur existence qu'aveo le lait de leur mère, et pendant lesquels, bien entendu, les allocations occasionnelles de thé léger ne sont pas décomptées comme aliments supplémentaires.

Pour juger la méthode de la F.M.S. de Greifswald, je rapporte ci-dessous la conclusion de Roos à la session de la Société allemande de médecine pour enfants (Lübeck, 10-13 septembre 1950);: " Par les deux méthodes de conservation à l'état brut (streptomycine et citrettes), l'effet bactériostatique ne suffit cependant occasionnellement pas ; c'est pourquoi elles exigent une stricte surveillance." 
La méthode de travail préconisée à Greifswald par Russ ne me paraît à aucun point de vue digne d'être imitée en raison de son incertitude, car elle ne répond pas aux sécurités exigées jusqu'à présent par les F.M.S. Les préjudices éventuels provoqués par la méthode de Greifswal pourraient bien retomber sur les F.M.S. et compromettre leur rènommée.

\title{
SUPPLEMENT TECHNIQUE
}

\section{LA PRÉPARATION DES ESTERS LACTIQUES PAR RÉACTION DU LACTATE D'AMIMONIUM AVEC LES ALCOOLS}

\author{
par \\ G. GÊNIN \\ Ingénieur E.P.C.
}

Des recherches entreprises il y a quelques mois par l'Eastern Regional Research Laboratory de Philadelphie [1] avaient montré que les sels d'ammonium ou ces sels substitués sont capables de réagir directement avec les alcools et de fournir les esters correspondants, l'autre produit de la réaction étant l'ammoniac ou une amine. Parmi les sels d'ammonium susceptibles d'être utilisés dans ce but, on avait observé que le lactate d'ammonium présente certains avantages. A la suite de cette première constatation, E. M. Filachione et E. J. Costello [2] ont procédé à une étude plus complète de l'emploi dè ce sel en vue de la préparation d'esters lactiques par réaction avec différents alcools. Nous donnons eidessous une analyse très complète de cette étude qui présente un certain intérêt, étant donné les applications possibles de ces esters comme plastifiants de nombreuse srésines synthétiques et matières plastiques.

Le lactate d'ammonium offre en effet d'importants avantages comme point de départ pour la fabrication des esters lactiques. Normalement, la préparation d'un ester lactique par le procédé classique comporte les opérations suivantes :

a) Fermentation d'hydrates de carbone en présence de carbonate de calcium, en vue de la préparation d'une solution de lactate de calcium;

b) Traitement par un acide de cette solution afin de mettre en liberté l'acide lactique, en même temps qu'il y a précipitation de sulfate de calcium ; 